\title{
Self-organisation of the human embryo in the absence of maternal tissues
}

\author{
Marta N. Shahbazi ${ }^{\# 1}$, Agnieszka Jedrusik ${ }^{\# 1}$, Sanna Vuoristo ${ }^{\# 1}$, Gaelle Recher ${ }^{1,5}$, Anna \\ Hupalowska $^{1}$, Virginia Bolton ${ }^{3}$, Norah N. M. Fogarty ${ }^{4}$, Alison Campbell ${ }^{2}$, Liani Devito ${ }^{3}$, \\ Dusko Ilic $^{3}$, Yakoub Khalaf ${ }^{3}$, Kathy K. Niakan ${ }^{4}$, Simon Fishel ${ }^{2}$, and Magdalena Zernicka- \\ Goetz ${ }^{1}$ \\ ${ }^{1}$ Mammalian Embryo and Stem Cell Group, University of Cambridge, Department of Physiology, \\ Development and Neuroscience; Downing Street, Cambridge, CB2 3DY, UK \\ ${ }^{2}$ CARE Fertility Group, John Webster House, 6 Lawrence Drive, Nottingham Business Park, \\ Nottingham, NG8 6PZ, UK \\ ${ }^{3}$ Faculty of Life Sciences and Medicine, King's College London, Women's Health Academic \\ Centre, Assisted Conception Unit, Guy's Hospital, Great Maze Pond, London SE1 9RT, UK \\ ${ }^{4}$ Human Embryo and Stem Cell Laboratory, Francis Crick Institute, Mill Hill Laboratory, London, \\ NW7 1AA, UK \\ \# These authors contributed equally to this work.
}

\begin{abstract}
Remodelling of the human embryo at implantation is indispensable for successful pregnancy. Yet it has remained mysterious because of the experimental hurdles that beset the study of this developmental phase. Here, we establish an in vitro system to culture human embryos through implantation stages in the absence of maternal tissues and reveal the key events of early human morphogenesis. These include segregation of the pluripotent embryonic and extra-embryonic lineages and morphogenetic re-arrangements leading to: generation of a bi-laminar disc, formation of a pro-amniotic cavity within the embryonic lineage, appearance of the prospective yolk sac, and trophoblast differentiation. Using human embryos and human pluripotent stem cells, we show that the reorganisation of the embryonic lineage is mediated by cellular polarisation leading to cavity formation. Together, our results indicate that the critical remodelling events at this stage of human development are embryo-autonomous highlighting the remarkable and unanticipated selforganising properties of human embryos.
\end{abstract}

Users may view, print, copy, and download text and data-mine the content in such documents, for the purposes of academic research, subject always to the full Conditions of use:http://www.nature.com/authors/editorial_policies/license.html\#terms

Correspondence should be addressed to M.Z-G. (mz205@ cam.ac.uk).

5 present address: Bioimaging and Optofluidics group, IOGS, CNRS \& University of Bordeaux. Rue Francois Mitterand, 33400

Talence, France.

Author contributions

M.N.S., A.J. and S.V. carried out all experiments and data analyses. G.R. analysed microscopy data and generated 3D reconstructions. A.H. prepared illustrations and contributed experimentally. N.F. and K.K.N. helped with human embryo cultures and contributed experimentally and L.D.G helped with human embryo cultures. A.C., S.F., D.I., Y.K. and K.K.N. oversaw and provided human embryos for these studies. M.Z-G. conceived the project and supervised the study. M.N.S. and M.Z-G. wrote the manuscript with help from all the authors. 


\section{Introduction}

The development of a human embryo from a fertilized egg begins with a series of cleavage divisions and morphogenetic rearrangements that lead to the formation of a free-floating blastocyst. This blastocyst comprises three distinct cell lineages: embryonic tissue (epiblast) and two extra-embryonic tissues (hypoblast and trophectoderm) that, after implantation into the uterus, will give rise to the yolk sac and placenta respectively. This pre-implantation period has been extensively studied using methods that pioneered in vitro fertilization (IVF)1, 2. However, on the 7th day of development, the human embryo must implant into the uterus of the mother to survive, establish the body plan and to generate the germ layers. The failure of an embryo to implant is a major cause of early pregnancy loss 3 and yet the cellular and molecular changes that take place in the human embryo at this stage remain unknown. This is because experiments in vivo are not feasible and there has been no system to culture human embryos ex vivo.

Monkey embryos have been used as a model for human embryo development 4,5 and the coculture of human blastocysts and endometrial cells6 has provided an experimental system to model post-implantation morphogenesis. However, the extent to which these systems recapitulate post-implantation development of the human embryo remains an open question. Moreover, the potential of human blastocysts for self-organisation has never been explored.

Here, to shed light on the early post-implantation phases of human development, we have adapted our recently established protocol for mouse embryos 7,8 . The culture system we have established allows human embryos to undergo the pre- to post-implantation transition in vitro, in the absence of any maternal tissues. By comparing human embryos developing in vitro and the Carnegie series of in vivo developing human embryos9, we have identified that the key hallmarks of human embryogenesis take place in the absence of any maternal tissues, uncovering the self-organising properties of human embryos at this stage.

\section{Results}

\section{Establishment of a method to culture human embryos through implantation stages in vitro}

In order to gain understanding of the developmental events undertaken by the implanting human embryo, we reasoned that we needed an in vitro culture system that would recapitulate these processes. To this end, we adapted the culture conditions we had previously established for mouse embryos at comparable stages7. Supernumerary preimplantation human embryos (at either cleavage or blastocysts stage) donated for this project were thawed and placed in culture medium to recover, before the zona pellucida was removed (Methods). Embryos were scored by morphological criteria and those showing abnormalities (fragmented blastomeres or developmentally arrested) were discarded. We first plated human blastocysts on optical grade dishes in $21 \% \mathrm{O}_{2}$ and in in vitro culture medium 1 (IVC1) which was replaced by IVC2 after 48h7 (Fig. 1a, b). Time-lapse microscopy revealed that human blastocysts cultured in IVC1 underwent a series of contractions and expansions reflecting collapse and subsequent enlargement of the blastocyst cavity (Supplementary Video 1), as observed in IVF clinics10. However, by the 
end of day 7, the blastocyst cavity completely collapsed and embryos attached to the dish (Fig. 1b-d). At this time the attached embryos started to grow and this growth continued until day 12-13 (Fig. 1c, d and Supplementary Video 2). We previously found that either human cord serum (HCS) or KnockOut Serum Replacement (KSR) in IVC2 permits periimplantation mouse development11. Here we show human embryos develop in medium supplemented with KSR up to day 13 (Fig. 1b-d). The experiment was stopped at this point because the internationally recognized ethical limit for human embryo culture is up to day 14 or to the first signs of development of the primitive streak 12 .

The trend in IVF clinics over recent years has been to culture pre-implantation human embryos in hypoxic conditions $\left(5 \% \mathrm{O}_{2}\right)$, as this favors embryo survival13 and inhibits differentiation in embryonic stem cells 14,15 . To investigate whether hypoxic conditions offer any advantage to human embryos cultured through implantation, we compared development of embryos cultured in $21 \% \mathrm{O}_{2}$ to ones cultured in $5 \% \mathrm{O}_{2}$ in terms of preservation of the pluripotent lineage. Initially, day 5-6 pre-implantation blastocysts had a group of inside pluripotent cells as indicated by the presence of the transcription factor OCT4 (Fig. 2a, b). However, after 4 days in culture, whereas $49 \%$ of embryos ( $\mathrm{N}=59)$ cultured in $21 \% \mathrm{O}_{2}$ maintained a cluster of epiblast cells showing expression of the pluripotency factor OCT4, none of the embryos cultured in hypoxic conditions $(\mathrm{N}=20)$ had OCT4-expressing cells (Supplementary Table 1). In contrast, cells of embryos in hypoxic conditions displayed signs of cell death (Fig. 2c). This suggests that preservation of the epiblast in vitro beyond day 7 might benefit from higher $\mathrm{O}_{2}$ concentrations possibly because of the increase in embryo size, which may decrease the $\mathrm{O}_{2}$ pressure in the core of the embryo. Hypoxia clearly has a complex influence on embryonic development and its effects need to be better understood in the intrauterine milieu to determine exactly how it affects both mouse and human embryogenesis 16 . As a result of these findings, here we cultured human embryos beyond day 7 in $21 \% \mathrm{O}_{2}$, which we show permits further development of the pluripotent lineage in vitro.

\section{Development of the embryonic and extra-embryonic lineages during human post- implantation morphogenesis in vitro}

To gain temporal understanding of the cellular and developmental events through early postimplantation development, we fixed and immunostained human embryos at different time points of culture. We used KSR to supplement the medium as it permits a more reproducible time course of development than HCS7. We have focused on development between days 7 and 11, since during these 5 days the embryo undergoes a major reorganisation that is necessary for subsequent gastrulation9. Shortly after attachment (day 7-8 of development), we observed a clear separation between embryonic (OCT4-expressing, epiblast) and extraembryonic (GATA6-expressing, hypoblast) cells, the respective progenitors for the fetus and yolk sac (Fig. 3a), whereas in the unattached blastocysts, this segregation of epiblast and hypoblast progenitors had not yet taken place (Fig. 2b). This contrasts to mouse embryos, where the epiblast and the hypoblast-equivalent (the primitive endoderm) segregate already before implantation 17; but agrees with observations in human embryos 18-22. We found that at day 7-8, the epiblast was formed by a cluster of about 20 OCT4-expressing cells surrounded by approximately 50 GATA6-expressing hypoblast cells (Fig. 3a, and 
Supplementary Table 2). By day 8-9, GATA6-expressing hypoblast cells preferentially localized to one side of the epiblast, as occurs in human embryos developing in vivo9. This spatial organisation was maintained throughout subsequent developmental stages (from day 9 to day 11) (Fig. 3a, Supplementary Fig. 1a and Supplementary Videos 3 and 4). By day 11, the number of OCT4-expressing epiblast cells had significantly increased to an average of 328 cells, whereas the number of GATA6-expressing hypoblast cells increased up to 79 (Supplementary Table 2).

To analyse development of trophectoderm, the progenitors of the placenta, we followed cytokeratin 7 (CK7), which is highly expressed in trophoblast derivatives23. We found that the trophectoderm cells surrounding the epiblast and hypoblast (Fig. 3b) presented a polarised epithelial phenotype, evident from the localization of the apical determinant PAR6 (Fig. 3c). From day 8 onwards, we noted the presence of CK7-expressing multinucleated cells in the outermost region of the embryo (Fig. 3d). To determine their exact position with respect to the epiblast-hypoblast cluster, we performed a 3D reconstruction of cellular shapes, based on the computational segmentation of membranes and nuclei (Fig. 3e). This revealed two trophectoderm subpopulations: cells in proximity to the epiblast-hypoblast bilayer had a single nucleus, whereas those in the periphery of the embryo were multinucleated and displayed characteristic lacunae (Fig. 3e and Supplementary Video 5). Based on the expression of CK7, their position and their cellular and nuclear shape, these two cell populations are likely to correspond to the cytotrophoblast and syncytiotrophoblast respectively, indicating that development post-implantation trophoblast-derivatives can be recapitulated in the absence of any maternal tissue. Together these results demonstrate that the development of all three lineages progresses as human embryos develop in vitro.

\section{Human epiblast polarisation and pro-amniotic cavity formation}

Next, we focused on the development of the epiblast since it undergoes its first major reorganisation at implantation. One of the hallmarks of epiblast transformation is the acquisition of a polarised phenotype and the formation of a lumen, the prospective proamniotic cavity9, 24, essential for the subsequent development of the body plan. However, the cellular mechanisms underlying lumen formation in humans currently remain unknown. While some reports in higher primates point towards cell death within the epiblast as the major driver of lumen formation 25 , alternative reports suggest folding of the epiblast26, or a change of polarity in epiblast cells4. Importantly, these hypotheses are based on the observations of Rhesus monkey embryos by electron microscopy as to date it has not been possible to definitively identify cell types and their features in human embryos at implantation. We found that at day 7-8 of development, the epiblast was a cluster of cells showing no signs of polarisation (Fig. 4a, Supplementary Fig. 1b and 2). However, by day 8-9, a group of OCT4-expressing epiblast cells became radially organised around a small central lumen. These cells were apico-basally polarised in $31 \%$ of the embryos (Supplementary Table 1), as determined by the polarised apical localization of actin and aPKC (the principal kinase of the apical Par polarity complex) (Fig. 4a and Supplementary Fig. 1b). The formation of a small lumen at the exact site of incipient apical polarisation indicates the onset of pro-amniotic cavity formation within the epiblast. Importantly, we did not observe any apoptotic cell, cellular debris or any other indication of cell death in the 
incipient pro-amniotic cavity (Supplementary Fig. 3). These results indicate that the epiblast becomes polarised by day 9 of in vitro development and suggest that apoptosis is not the mechanism driving lumenogenesis in human embryos.

\section{Human bi-laminar disc formation}

The next expected remodelling event is the formation of the amniotic epithelium and the epiblast disc. In contrast to the mouse, in which the amnion is formed at gastrulation, the amnion is expected to form in humans shortly after implantation with those epiblast cells adjacent to the hypoblast acquiring a columnar shape, and those in contact with cytotrophoblast differentiating into squamous and flat amniotic epithelium. At this stage, both tissues line the pro-amniotic cavity 24 . We observed that as development of embryos progressed in vitro, the epiblast and the pro-amniotic cavity expanded in size (Fig. 4a and b, Supplementary Fig. 4, Supplementary Table 2 and Supplementary Video 6) indicative of embryo growth. At day 10-11 of development, we could detect two morphologically distinct groups of OCT4-expressing cells. 3D reconstructions of cellular shapes revealed that OCT4expressing cells located near the hypoblast had a columnar morphology whereas oppositefacing OCT4-expressing cells had a distinct squamous and flat shape (Fig. 4c and Supplementary Video 7). Moreover, these flat OCT4-expressing cells were in close proximity to cells with a higher nuclear volume (on average 2.5 times bigger than epiblast cells), a feature characteristic of trophoblast cells. These observations suggest that the two types of OCT4-expressing cells may represent the epiblast disc and the prospective amniotic epithelium.

\section{Human prospective yolk sac formation}

The next expected event of human embryo development is the reorganisation of hypoblast cells to give rise to a second cavity, the primary yolk sac9, 27, which will provide the blood supply to the developing fetus. Remarkably, we found that human embryos developing in vitro established a second cavity at day 11 of development (Supplementary Video 8). This cavity was localized below the epiblast and was surrounded by GATA6-expressing cells, highlighting its hypoblast origin (Fig. 4d, e and Supplementary Video 9). Thus, both expression of characteristic lineage markers as well as morphological features suggest that formation of the prospective yolk sac can be also recapitulated in human embryos developing in vitro (Fig. 4f).

\section{Human pluripotent stem cells (hPSCs) recapitulate the events of polarisation and lumenogenesis}

Since the above results revealed that epiblast polarisation and lumen formation are the first features of epiblast morphogenesis after implantation, we next investigated the molecular pathways that could be responsible for this critical remodelling. Given the ethical restrictions and the limited number of human embryos available for functional studies, we turned to human embryonic stem cells (hESCs) and human induced pluripotent stem cells (hiPSCs) as alternative models. In order first to determine whether the extracellular matrix (ECM) participates in inducing epiblast polarisation and lumen formation, we cultured individual hESCs in 3D matrigel. We found that surrounding cells with ECM enabled lumen formation by $24 \mathrm{~h}$ after cell plating, and so it was sufficient to have two sister cells from a single cell 
division for this to take place (Fig. 5a), in agreement with other recent observations28. As the hESCs continued to divide, they self-organised around a central lumen. These hESCsorganoids were apico-basally polarised, as demonstrated by the polarised localization of aPKC (Fig. 5a), PAR6 (Fig. 5b), centrosomes (Fig. 5c) and the Golgi (Fig. 5d). They retained expression of pluripotency markers such as OCT4 (Fig. 5a), mimicking the preservation of OCT4 in the epiblast of in vitro cultured human embryos (Fig. 3). To confirm these results we next plated hiPSCs into 3D matrigel as we did with hESCs. We found that hiPSCs also polarised and began to form lumens between two cells, while retaining OCT4 expression (Fig. 6a). Lumens formed on the apical side of polarised cells that displayed apical microvilli and a polarised distribution of aPKC, PAR6 and the Golgi (Fig. 6a-c). This polarised organisation was preserved in the presence of the ROCK inhibitor Y-27632 (Fig. 6d), commonly used to avoid cell death at the time of plating of hPSCs. We could also detect lumen-directed secretion of the anti-adhesive protein Podocalyxin (PODXL), implicated in lumen formation through charge-repulsion29, 30 (Fig. 6e, f). The observation that hPSC-organoids develop lumens between two cells as they become polarised, suggests that lumen formation is not a consequence of cell death. To test this possibility directly, we treated hiPSCs with a caspase 3 inhibitor to prevent cell death8 and found that polarisation and lumen formation were not affected (Fig. 6g, h). Taken together our results indicate that lumen formation is a consequence of cellular polarisation and not cell death and lead us to suggest that similar molecular pathways may drive lumenogenesis in human embryos soon after implantation.

\section{Discussion}

Implantation is a milestone in human development as this is the time when the embryo undergoes major remodelling, which is absolutely required for correct gastrulation and therefore successful body formation and pregnancy outcome. However, to date, the absence of an in vitro culture system to visualise and model this critical developmental transition has severely limited our understanding of human embryogenesis. Here, we have established a system for the in vitro culture of human embryos that offers a unique opportunity to understand human development after implantation. This system has allowed us to uncover the major morphogenetic events that normally occur after human embryo implantation including: (i) epiblast and hypoblast segregation; (ii) epiblast polarisation; (iii) formation of the pro-amniotic cavity and the bi-laminar disc; (iv) appearance of the prospective amniotic ectoderm; (v) appearance of yolk sac; and (vi) differentiation of the trophoblast into cytotrophoblast and syncytiotrophoblast (Fig. 4f). Although this system may not be able to fully recapitulate all the complex aspects of human embryogenesis in vivo, it has allowed us to reveal a remarkable self-organising capacity of human blastocysts that has been previously unknown. The next stage in human development corresponds to primitive streak formation but for ethical reasons we are obliged to stop our cultures at day 14 of development or before the primitive streak formation12. Thus, our studies have focused on the key events of pre-gastrulation stages.

Our results indicate that the formation of the pro-amniotic cavity in the human embryo is not triggered by apoptosis, as hypothesised 25 , but by the epiblast's polarisation, as proposed in monkey embryos4. Because of the limitations to perform functional studies in human 
embryos, we have used hPSCs as an alternative model to test this hypothesis, and demonstrate that the ECM participates in inducing cell polarisation and lumen formation via polarised secretion (hollowing) and not programmed cell death (cavitation). This is similar to the mechanism of epiblast polarisation we have recently found to operate in mouse embryos8. Thus, remarkably, despite the significant differences in embryo morphology between these different mammalian species, the initial steps of post-implantation embryogenesis are evolutionarily conserved.

One of the most intriguing aspects of human implantation development is the differentiation of the epiblast into the epiblast disc and the amniotic epithelium24. Interestingly, we could not recapitulate formation of the amniotic epithelium in our 3D hPSC culture possibly because this developmental process requires interaction with extra-embryonic tissues present in the whole embryo. It will be of interest to determine the signalling pathways required for modelling amnion formation in vitro. Indeed, we anticipate that future studies using the in vitro culture systems we report here for both human embryos and hPSCs will shed new light on the cellular and molecular mechanisms of this mysterious and yet critical stage of human development, which will be of basic and clinical importance.

\section{Methods}

\section{Ethics Statement}

Human embryo experiments were performed in three different groups under three different licenses obtained from the Human Fertilization and Embryology Authority (HFEA):

- $\quad$ License reference R0193-1-a (University of Cambridge): Cryopreserved human embryos were donated to this project, entitled "Filming of human implantation in vitro", after an informed consent of couples undergoing IVF treatments. Prior to giving consent persons donating embryos were provided with all the necessary information about the research project and conditions that apply within the license and HFEA Code of Practice. In addition, an independent ethic approval was obtained from the "Human Biology Research Ethics Committee" of the University of Cambridge.

- $\quad$ License reference R0075 (Kings College London, Guy's Hospital): Informed consent was obtained from all subjects and the experiments conformed to the principles set out in the WMA Declaration of Helsinki and the NIH Belmont Report. No financial inducements are offered for donation. This project has also a local ethical approval (UK National Health Service Research Ethics Committee Reference: 06/Q0702/90).

- $\quad$ License reference R0162 (The Francis Crick Institute): Informed consent was obtained from all couples that donated spare embryos following IVF treatment. Prior to giving consent persons donating embryos were provided with all of the necessary information about the research project, an opportunity to receive counseling and the conditions that apply within the license and the HFEA Code of Practice. No financial inducements are offered for donation. This 
project also has ethical approval from the UK National Health Services Research Ethics Committee Reference: 04/Q0108/99.

In the three locations involved in this project, all the experiments on human embryos were performed under the HFEA Codes of Practice and the Human Fertilization and Embryology Act 1990 practices. Human embryos were not maintained in culture past 14 days or appearance of the primitive streak.

Human embryonic stem cell work complies the regulations of the and UK Code of Practice for the Use of Human Stem Cell Lines and UK Stem Cell Bank Steering committee. An ethical approval for this project was obtained from the UK Stem Cell Steering Committee.

\section{Human embryo thawing and zona pellucida removal}

Human blastocysts (day 5 and day 6), were thawed using Kitazato Thawing Media Kit VT802 (91182; Kitazato Dibimed; Shizuoka, Japan), or Quinn's Advantage® Thaw Kit (ART-8016; LifeGlobal®Group; Guelph, Canada) depending on the protocol used for freezing and following the manufacturer's instructions. Cleavage stage embryos (day 1, day 2 and day 3) were thawed using Embryo Thawing Pack (10984010; Origio; Malov, Denmark) and cultured in drops of human embryo culture medium (LGGG-020; LifeGlobal $®$ Group; Guelph, Canada) supplemented with $10 \%$ (vol/vol) protein supplement (LPGS-605; LifeGlobal®Group; Guelph, Canada) under mineral oil (9305; Irvine Scientific; California, US) until blastocyst stage. The zona pellucida of each blastocyst-stage embryo was removed by brief exposure to Acidic Tyrode's solution (T1788; Sigma-Aldrich; St. Louis, US), embryos were washed in the human embryo culture medium (LGGG-020; LifeGlobal@Group; Guelph, Canada), or SAGE 1-Step ${ }^{\mathrm{TM}}$ (67010060A; LifeGlobal@Group; Guelph, Canada) and transferred to in vitro culture medium (see below).

\section{Human embryo in vitro culture from pre- to post-implantation stage}

The in vitro culture procedure is illustrated in Figure 1a. Day 5-6 human blastocysts devoid of zona pellucida were cultured on 8-well IbiTreat $\mu$-plates (IB-80826; Ibidi GmbH; Martinsried, Germany) with in vitro culture media at $37^{\circ} \mathrm{C}$, in $21 \% \mathrm{O}_{2} / 5 \% \mathrm{CO}_{2}$ or when indicated in $5 \% \mathrm{O}_{2} / 5 \% \mathrm{CO}_{2}$. For the first $48 \mathrm{~h}$ the embryos were cultured in in vitro culture medium 1 (IVC1; see below)7. On the in vitro culture day 2 (embryo day 7-8 post fertilization), $50 \%$ of the culture medium was replaced with the in vitro culture medium 2 (IVC2; see below)7. From in vitro culture day 3 on, IVC2 medium was used and medium was refreshed on daily basis. Two alternative IVC2 compositions were used. The detailed composition of the media used is outlined below:

- $\quad$ IVC1: Advanced DMEM/F12 (12634-010; Thermo Fischer Scientific; Waltham, US) supplemented with $20 \%$ (vol/vol) heat-inactivated FBS (Produced in house, Stem Cell Institute, the University of Cambridge, UK; or 35-015-CV; Corning; New York, US), 2mM L-Glutamine (25030; Thermo Fisher Scientific; Waltham, US), penicillin (25 units/ml)/Streptomycin (25 $\mu \mathrm{g} / \mathrm{ml}$ ) (15070-063; Thermo Fischer Scientific; Waltham, US), 1X ITS-X (10 $\mathrm{mg} / \mathrm{L}$ insulin , $5.5 \mathrm{mg} / \mathrm{L}$ transferrin, $0.0067 \mathrm{mg} / \mathrm{L}$ sodium selenite, $2 \mathrm{mg} / \mathrm{L}$ etholamine; 51500-056; Thermo Fisher Scientific; Waltham, US), 8 nM $\beta$ - 
estradiol (E8875; Sigma-Aldrich; St. Louis, US), $200 \mathrm{ng} / \mathrm{ml}$ progesterone (P0130; Sigma-Aldrich; St. Louis, US) and $25 \mu \mathrm{M} \mathrm{N}$-acetyl- ${ }_{-}$-cysteine (A7250; Sigma-Aldrich; St. Louis, US).

Defined IVC2: Advanced DMEM/F12, supplemented with 30\% (vol/vol) KnockOut Serum Replacement (10828010; Thermo Fisher Scientific; Waltham, US), $2 \mathrm{mM}$ L-Glutamine, Penicillin ( 25 units/ml)/Streptomycin (25 $\mu \mathrm{g} / \mathrm{ml}), 1 \mathrm{X}$ ITS-X, $8 \mathrm{nM} \beta$-estradiol, $200 \mathrm{nG} / \mathrm{mL}$ progesterone, and $25 \mu \mathrm{M} N$ acetyl-L-cysteine. The same catalogue numbers and suppliers apply as above.

A step-by-step protocol of the in vitro culture of human embryos through implantation stages can be found at Nature Protocol Exchange34.

\section{Human embryo time-lapse imaging}

Time-lapse phase contrast images were acquired using spinning disc confocal microscopy (3i Intelligent Imaging Innovations) and a Zeiss 0.55 NA 20X (EC PlnN) objective; and wide field microscopy ( $3 \mathrm{i}$ Intelligent Imaging Innovations) and a Zeiss Plan-Apo Chromat 0.75 NA 20X objective. The embryos were imaged in humidified $21 \% \mathrm{O}_{2}$. Z-range of 120 $\mu \mathrm{m}$ with $5 \mu \mathrm{m}$ intervals were scanned in every $30 \mathrm{~min}$. The images were processed using Fiji (http://fiji.sc/Fiji).

\section{Immunofluorescence of human embryos}

Embryos were fixed with $4 \%$ paraformaldehyde (PFA) for $20 \mathrm{~min}$ at room temperature, washed with phosphate buffered saline (PBS), and permeabilized by $0.5 \%$ Triton X-100 in PBS at room temperature for 20 min. Embryos were blocked with 10\% FBS (Produced in house, Stem Cell Institute, Cambridge, UK)/3\% (w/vol) bovine serum albumin (A3311; Sigma-Aldrich, St. Louis, US) in PBS, at room temperature for $4 \mathrm{hrs}$. The primary antibodies mouse monoclonal anti-Oct3/4 (C-10; sc-5279; Santa Cruz Biotechnology; Dallas, US; 1:200), rabbit polyclonal anti-aPKC (C-20; Sc-216; Santa Cruz Biotechnology; Dallas, US; 1:200), goat monoclonal anti-GATA6 (clone 222228; mab1700; R\&D Systems; Minneapolis, US; 1:200), rabbit polyclonal anti-PARD6B (M-64; sc-67393; Santa Cruz Biotechnology; Dallas, US; 1:200) and mouse monoclonal anti-Cytokeratin 7 (OVTL 12-30; sc-52322; Santa Cruz Biotechnology; Dallas, US; 1:200) were diluted in 3\% (w/vol) bovine serum albumin (as above)/0.1\% Tween20 (P9416; Sigma Aldrich; St.Louis, US) in PBS and incubated at $4{ }^{\circ} \mathrm{C}$ for over night. Samples were incubated with the following fluorescenceconjugated secondary antibodies: donkey anti-mouse AlexaFluor ${ }^{\circledR 568}$, donkey anti-rabbit AlexaFluor®647, donkey anti-goat AlexaFluor ${ }^{\circledR} 488$ from Thermo Fisher Scientific; Waltham, US. All secondary antibodies were diluted 1:500 in 3\% (w/vol) bovine serum albumin (as above)/0.1\% Tween20 (P9416; Sigma Aldrich; St.Louis, US) in PBS. F-actin was stained with AlexaFluor®488 Phalloidin (A12379; Thermo Fisher Scientific; Waltham, US; 1:200) and nuclei were stained with DAPI (D3571; Thermo Fisher Scientific; Waltham, US; $1 ; 1000)$.

\section{Human pluripotent stem cell culture}

hESC line H9 and hiPSC line FSPS 13B were kindly provided by Prof. Ludovic Vallier (Stem Cell Institute, Anne McLaren Laboratory, Cambridge, UK). The hiPSC line 
SC101A-1 (System Biosciences)31 was kindly provided by Dr. Madeline Lancaster (MRC Laboratory of Molecular Biology, Cambridge, UK). These hPSCs were maintained on Geltrex (A15696-01; Thermo Fisher Scientific; Waltham, US) -coated culture plates in complete serum-free defined hPSC culture media, either Essential 8 (E8; A1517001; Thermo Fisher Scientific; Waltham, US) or mTeSR ${ }^{\mathrm{TM}} 1$ (05850; Stem Cell Technologies; Vancouver, Canada). Authentication of the cell lines was done based on pluripotent gene expression. No cell lines used in this study were found in the database of commonly misidentified cell lines that is maintained by ICLAC and NCBI Biosample and they were not routinely tested for mycoplasma.

\section{D culture of hPSCs}

hPSCs were suspended as single cells using StemPro Accutase Cell Dissociation Reagent (A11105-1; Thermo Fisher Scientific; Waltham, US) and plated following a 3D on top protocol as previously described32. Briefly, IbiTreat $\mu$-plates (IB-80826; Ibidi GmbH; Martinsried, Germany) were coated with Matrigel Basement Membrane Growth Factor Reduced (354230; Corning; New York, US) and $2 \times 10^{4}$ cells were plated in either E8 or mTeSR ${ }^{\mathrm{TM}} 1$ medium. After 5 min and upon attachment of the cells to the matrigel layer, the medium was removed and either fresh $\mathrm{E} 8$ or $\mathrm{mTeSR}^{\mathrm{TM}} 1$ medium supplemented with 5\% Matrigel was added on top. When indicated, $10 \mu \mathrm{M}$ ROCK inhibitor Y-27632 (Y0503; Sigma-Aldrich; St. Louis, US) or 10/20 $\mu$ M Caspase 3 inhibitor Z-DEVD-FMK (1143-5; BioVision; Milpitas, US) were added to the medium.

A step-by-step protocol of the 3D culture of hPSCs can be found at Nature Protocol Exchange34.

\section{Immunofluorescence of 3D cultured hPSCs}

hPSCs embedded in Matrigel were fixed with either 4\% PFA at room temperature or icecold methanol at $-20^{\circ} \mathrm{C}$ for $15 \mathrm{~min}$. The samples were permeabilized with $0.3 \% \mathrm{Tx} 100-0.1$ $\mathrm{M}$ glycine in PBS for $20 \mathrm{~min}$. The primary antibodies mouse monoclonal anti-Oct3/4 (as above, 1:200), rabbit polyclonal anti-aPKC (as above, 1:200), rabbit polyclonal antiPARD6B (as above, 1:200), mouse monoclonal anti-GM130 (clone 35/GM130; 610822; BD Biosciences; San Jose, US; 1:200), mouse monoclonal anti- $\gamma$-tubulin (clone GTU-88; T5326; Sigma, St.Louis, US; 1:500) and mouse monoclonal anti-PODXL (clone 222328; MAB1658; R\&D Systems; Minneapolis, US; 1:200) were diluted in blocking buffer (1\% Bovine Serum Albumin - A3311; Sigma-Aldrich; St. Louis, US - and 0.1\% Tween) and incubated at $4^{\circ} \mathrm{C}$ over night. Samples were washed three times with PBS-0.1\% Tween and incubated with AlexaFluor®-conjugated secondary antibodies (as above).

\section{Imaging}

The images of the fixed samples were acquired using inverted SP5 confocal microscope (Leica Microsystems, Manheim, Germany) and Leica 1.3 NA 40X oil (HC PL APO), Leica 1.4 NA 63X oil (HC PL APO) and Leica 1.3 NA 63x glycerol (HCX PL APO) objectives. The images were processed using Fiji (http://fiji.sc), Chimera (https://www.cgl.ucsf.edu/ chimera/) and MovIT visualization software (BioEmergences, http:// bioemergences.iscpif.fr/)33. 


\section{Statistics and reproducibility}

The exact number of embryos analysed in the different conditions, as well as the number of embryos that displayed a polarized epiblast is indicated in Supplementary Table 1.

No statistical method was used to predetermine sample size. Given the nature of the work (mostly descriptive) no statistical comparisons were done between different embryos. Embryos that showed abnormalities (fragmented blastomeres or developmentally arrested) were excluded from the analysis. The investigators were not blinded to allocation during experiments and outcome assessment.

Figure 1 involved the assessment of 5 embryos collected across 3 independent experiments. Out of these 5 embryos 3 showed a correct development.

Figure 2 involved the assessment of 20 embryos cultured in 5\% $\mathrm{O}_{2}$ and collected across 3 independent experiment. All 20 embryos lacked the pluripotent lineage (phenotype shown in the image). Embryos were randomly assigned to either $5 \% \mathrm{O}_{2}$ or $21 \% \mathrm{O}_{2}$.

Figures 3 and 4 involved the assessment of 59 embryos collected across 6 independent experiments. Briefly, 29 embryos preserved a cluster of pluripotent cells; and out of these 29 embryos, 9 had an organized epiblast.

Figures 5 and 6 involved the assessment of a minimum of $10 \mathrm{hPSC}$ organoids per panel and condition. These were collected across a minimum of 2 independent experiments per panel.

Qualitative data are presented in the form of a contingency table (Fig. 6h). This data was analyzed using a Fisher's exact test.

Quantitative data are presented as mean \pm s.e.m. The normality of the data was analyzed with a D'Agostino\&Pearson normality test. Data presented in Fig. 5b displayed a normal distribution with significantly different variances. This was analyzed using an unpaired twotailed Student's t test with a Welch's correction.

\section{Supplementary Material}

Refer to Web version on PubMed Central for supplementary material.

\section{Acknowledgments}

\footnotetext{
We are grateful to the patients donating their embryos and colleagues in Prof. M.Z-G. lab, Ms. C. Lee (Gurdon Institute), and embryologists at the CARE, Bourn Hall (Kay Elder and Phil Snell) and Kings College Guy's Hospital IVF clinics for help and discussions. We thank Prof. P. Braude, Prof. D. Glover and Dr. C. Ogilvie for insightful discussion and Dr. I. Bedzhov for help in a pilot experiment. This work was supported by the Wellcome Trust grant to M.Z-G. Work in Dr. K.K.N lab was supported by The Francis Crick Institute, which receives its core funding from Cancer Research UK, the Medical Research Council and the Wellcome Trust. Dr. M.N.S. was initially supported by a Ramon Areces Spanish Foundation Fellowship, and subsequently by an EMBO Postdoctoral Fellowship. Dr. S.V was supported by a Post Doc Pool Grant from the Finnish Cultural Foundation. Dr. GR was supported by a Newton Fellowship.
} 


\section{References}

1. Edwards RG, Bavister BD, Steptoe PC. Early stages of fertilization in vitro of human oocytes matured in vitro. Nature. 1969; 221:632-635. [PubMed: 4886881]

2. Edwards RG, Steptoe PC, Purdy JM. Fertilization and cleavage in vitro of preovulator human oocytes. Nature. 1970; 227:1307-1309. [PubMed: 4916973]

3. Koot YE, Teklenburg G, Salker MS, Brosens JJ, Macklon NS. Molecular aspects of implantation failure. Biochim Biophys Acta. 2012; 1822:1943-1950. [PubMed: 22683339]

4. Enders AC, Schlafke S, Hendrickx AG. Differentiation of the embryonic disc, amnion, and yolk sac in the rhesus monkey. Am J Anat. 1986; 177:161-185. [PubMed: 3788819]

5. Pera MF, Trounson AO. Human embryonic stem cells: prospects for development. Development. 2004; 131:5515-5525. [PubMed: 15509763]

6. Weimar CH, Post Uiterweer ED, Teklenburg G, Heijnen CJ, Macklon NS. In-vitro model systems for the study of human embryo-endometrium interactions. Reprod Biomed Online. 2013; $27: 461-$ 476. [PubMed: 24055530]

7. Bedzhov I, Leung CY, Bialecka M, Zernicka-Goetz M. In vitro culture of mouse blastocysts beyond the implantation stages. Nat Protoc. 2014; 9:2732-2739. [PubMed: 25356584]

8. Bedzhov I, Zernicka-Goetz M. Self-organizing properties of mouse pluripotent cells initiate morphogenesis upon implantation. Cell. 2014; 156:1032-1044. [PubMed: 24529478]

9. Hertig AT, Rock J, Adams EC. A description of 34 human ova within the first 17 days of development. Am J Anat. 1956; 98:435-493. [PubMed: 13362122]

10. Hur YS, et al. Effect of artificial shrinkage on clinical outcome in fresh blastocyst transfer cycles. Clin Exp Reprod Med. 2011; 38:87-92. [PubMed: 22384424]

11. Morris SA, et al. Dynamics of anterior-posterior axis formation in the developing mouse embryo. Nat Commun. 2012; 3:673. [PubMed: 22334076]

12. Pera MF, et al. What if stem cells turn into embryos in a dish? Nat Methods. 2015; 12:917-919. [PubMed: 26418764]

13. Gardner DK. The impact of physiological oxygen during culture, and vitrification for cryopreservation, on the outcome of extended culture in human IVF. Reprod Biomed Online. 2015

14. Covello KL, et al. HIF-2alpha regulates Oct-4: effects of hypoxia on stem cell function, embryonic development, and tumor growth. Genes Dev. 2006; 20:557-570. [PubMed: 16510872]

15. Ezashi T, Das P, Roberts RM. Low O2 tensions and the prevention of differentiation of hES cells. Proc Natl Acad Sci U S A. 2005; 102:4783-4788. [PubMed: 15772165]

16. Rivera-Perez JA, Jones V, Tam PP. Culture of whole mouse embryos at early postimplantation to organogenesis stages: developmental staging and methods. Methods Enzymol. 2010; 476:185-203. [PubMed: 20691867]

17. Bedzhov I, Graham SJ, Leung CY, Zernicka-Goetz M. Developmental plasticity, cell fate specification and morphogenesis in the early mouse embryo. Philos Trans R Soc Lond B Biol Sci. $2014 ; 369$

18. Roode M, et al. Human hypoblast formation is not dependent on FGF signalling. Dev Biol. 2012; 361:358-363. [PubMed: 22079695]

19. Teklenburg G, et al. Cell lineage specific distribution of H3K27 trimethylation accumulation in an in vitro model for human implantation. PLoS One. 2012; 7:e32701. [PubMed: 22412909]

20. Niakan KK, Eggan K. Analysis of human embryos from zygote to blastocyst reveals distinct gene expression patterns relative to the mouse. Dev Biol. 2013; 375:54-64. [PubMed: 23261930]

21. O'Leary T, et al. Tracking the progression of the human inner cell mass during embryonic stem cell derivation. Nat Biotechnol. 2012; 30:278-282. [PubMed: 22371082]

22. Niakan KK, Han J, Pedersen RA, Simon C, Pera RA. Human pre-implantation embryo development. Development. 2012; 139:829-841. [PubMed: 22318624]

23. Haigh T, Chen C, Jones CJ, Aplin JD. Studies of mesenchymal cells from 1st trimester human placenta: expression of cytokeratin outside the trophoblast lineage. Placenta. 1999; 20:615-625. [PubMed: 10527816] 
24. Dobreva MP, Pereira PN, Deprest J, Zwijsen A. On the origin of amniotic stem cells: of mice and men. Int J Dev Biol. 2010; 54:761-777. [PubMed: 20446274]

25. Hill JP. The developmental history of the primates. Philos Trans R Soc Lond B Biol Sci. 1932; 221:45-178.

26. Luckett WP. The development of primordial and definitive amniotic cavities in early Rhesus monkey and human embryos. Am J Anat. 1975; 144:149-167. [PubMed: 810017]

27. Palis J, Yoder MC. Yolk-sac hematopoiesis: the first blood cells of mouse and man. Exp Hematol. 2001; 29:927-936. [PubMed: 11495698]

28. Taniguchi K, et al. Lumen Formation Is an Intrinsic Property of Isolated Human Pluripotent Stem Cells. Stem Cell Reports. 2015; 5:954-962. [PubMed: 26626176]

29. Bryant DM, et al. A molecular switch for the orientation of epithelial cell polarization. Dev Cell. 2014; 31:171-187. [PubMed: 25307480]

30. Bryant DM, Mostov KE. From cells to organs: building polarized tissue. Nat Rev Mol Cell Biol. 2008; 9:887-901. [PubMed: 18946477]

31. Lancaster MA, et al. Cerebral organoids model human brain development and microcephaly. Nature. 2013; 501:373-379. [PubMed: 23995685]

32. Lee GY, Kenny PA, Lee EH, Bissell MJ. Three-dimensional culture models of normal and malignant breast epithelial cells. Nat Methods. 2007; 4:359-365. [PubMed: 17396127]

33. Faure E, et al. A workflow to process $3 \mathrm{D}+$ time microscopy images of developing organisms and reconstruct their cell lineage. Nat Commun. 2016; 7:8674. [PubMed: 26912388]

34. Vuoristo S, Jedrusik A, Shahbazi MN, Zernicka-Goetz M. Culture of human embryos through implantation stages in vitro. Protocol Exchange. 2016; doi: 10.1038/protex.2016.017 


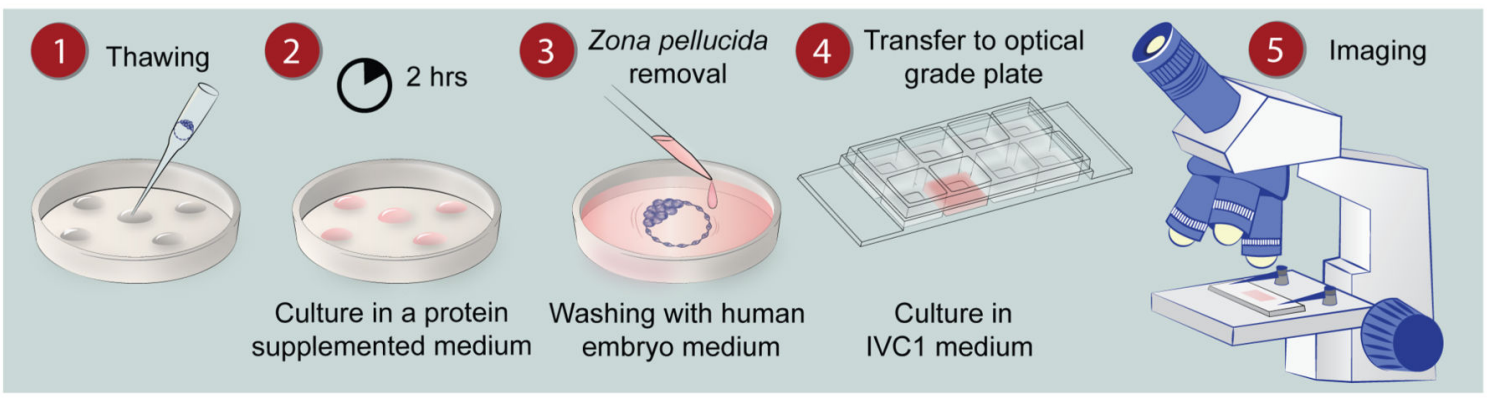

b

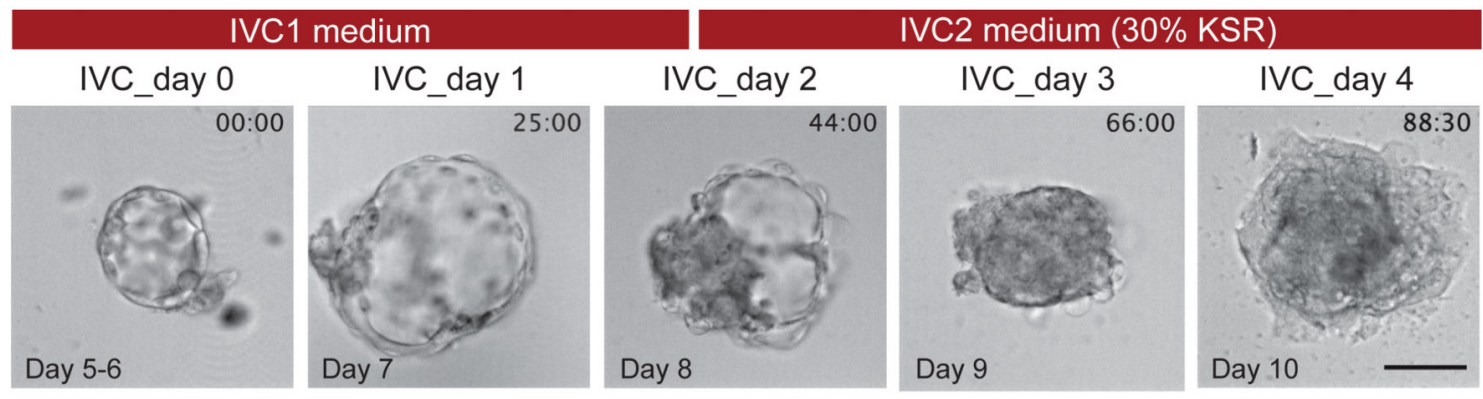

C

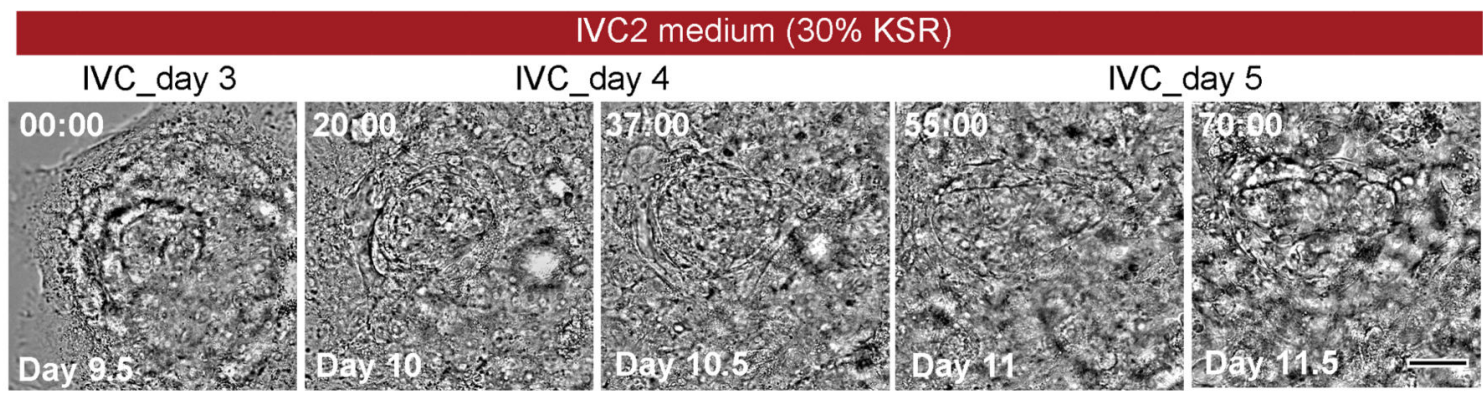

d
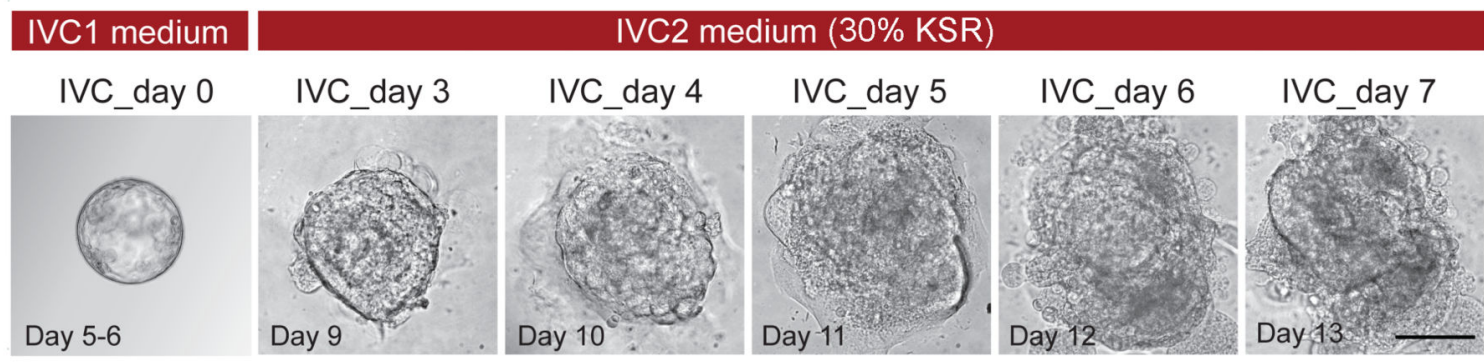

Figure 1. Establishment of an in vitro system to study human implantation and early postimplantation morphogenesis.

Human embryos were thawed and cultured until the blastocyst stage (day 5-6 of development). The zona pellucida was removed and embryos were transferred to plates in IVC1 medium for imaging. On the second day of culture, medium was changed for IVC2 with $30 \%$ KnockOut Serum Replacement (KSR) (b, c) or $20 \%$ human cord serum (HCS) (d). Shown are representative bright field images of human blastocysts developing in vitro 
until day 12-13. All scale bars, $100 \mu \mathrm{m}$. These data involved the assessment of a total of 5 embryos collected across 3 experiments, out of which 3 showed a correct development. 
a

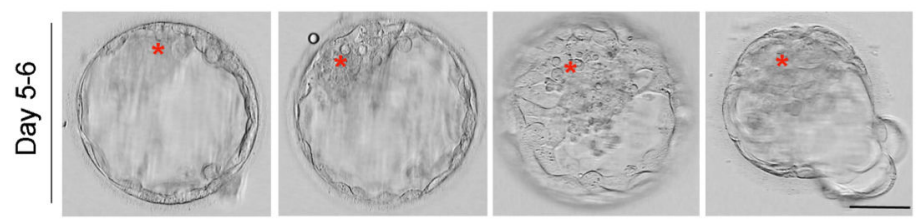

b

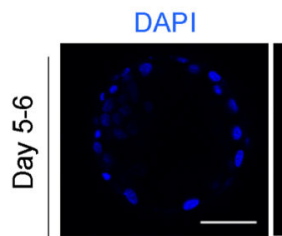

GATA6

OCT4

GATA6/OCT4

DAPI
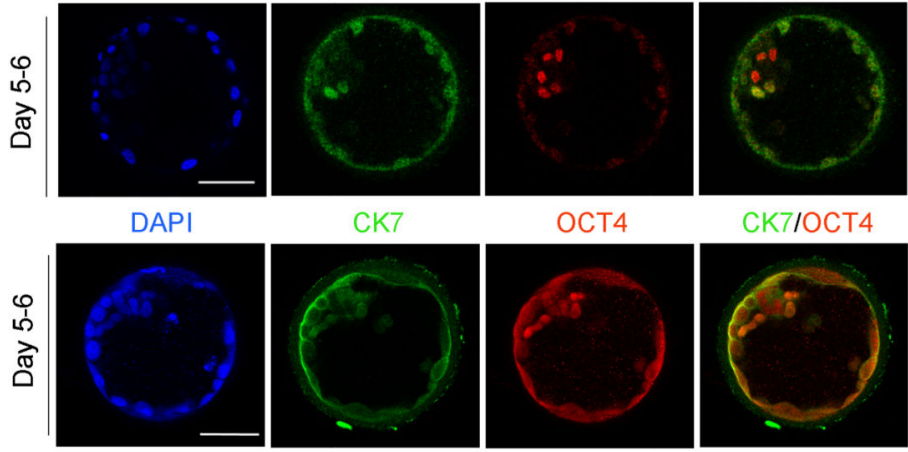

CK7

OCT4

CK7IOCT4
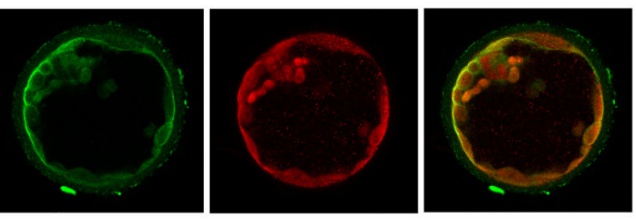

C

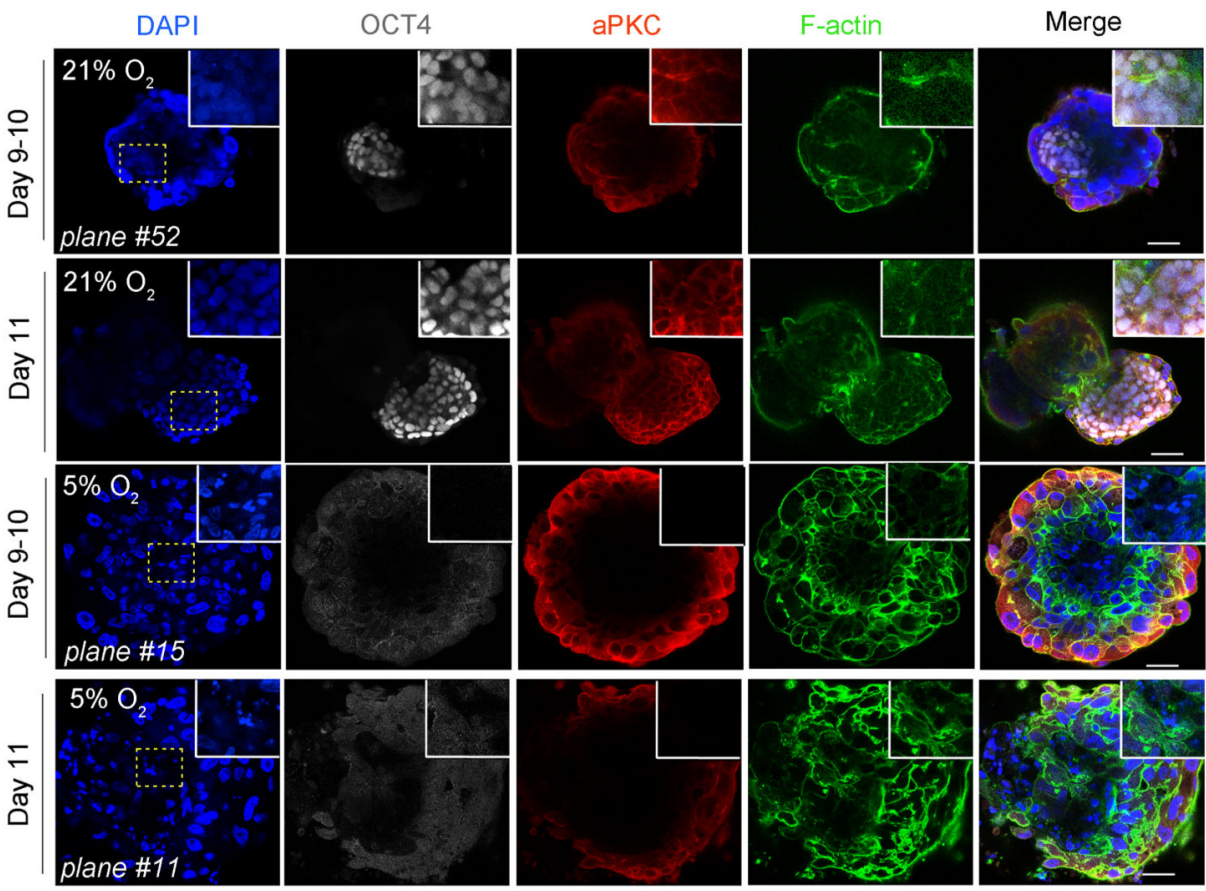

Figure 2. Preservation of the pluripotent lineage in human embryos cultured through implantation stages in vitro.

a, Bright field images of day 5-6 blastocysts. Asterisks indicate the inner cell mass. b, Day 5-6 blastocysts were fixed and immunostained for lineage markers. Representative confocal Z sections of human blastocysts stained for OCT4 and GATA6, and OCT4 and CK7. c, Human embryos cultured in either $21 \%$ or $5 \% \mathrm{O}_{2}$ were analysed at indicated time points. Representative confocal Z sections of human embryos stained for OCT4, aPKC and F-actin. Note the absence of OCT4-expressing cells and the presence of fragmented nuclei in the 
embryos cultured in 5\% $\mathrm{O}_{2}$. All scale bar, $50 \mu \mathrm{m}$. These data involved the assessment of a total of 59 embryos in $21 \% \mathrm{O}_{2}$ (out of which 29 embryos preserved the epiblast) and 20 embryos in $5 \% \mathrm{O}_{2}$ (out of which none preserved the epiblast) collected across 3 experiments. 
a

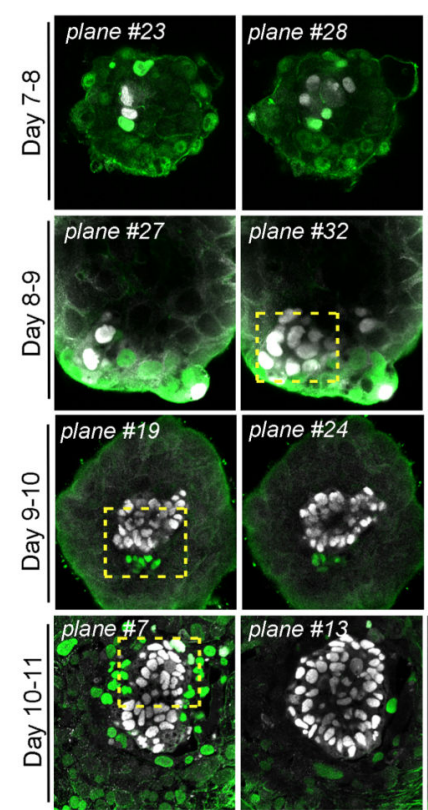

b

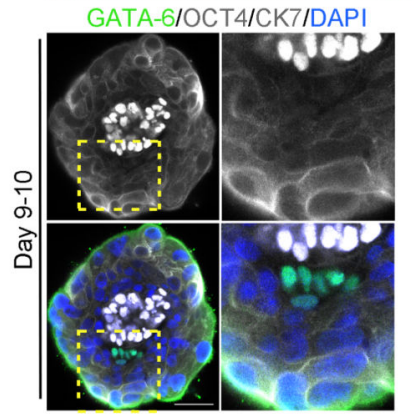

d
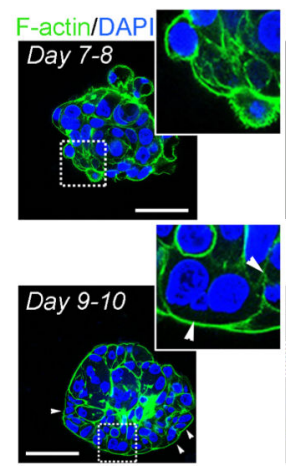

GATA-6/OCT4
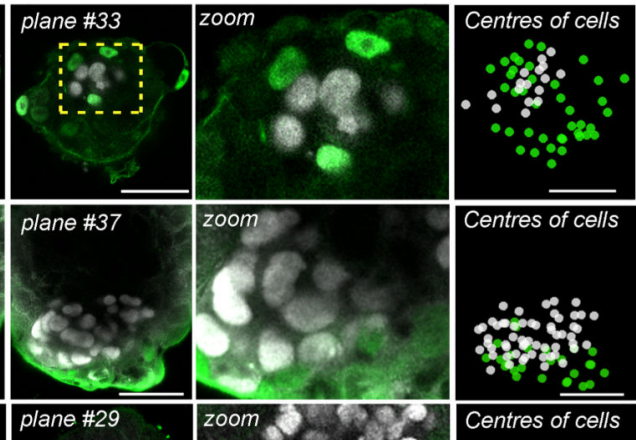

plane \#29
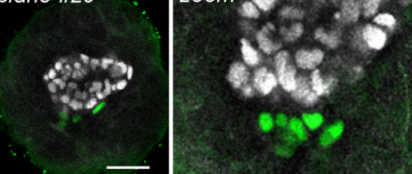

Centres of cells

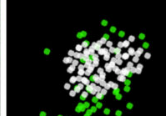

$\because \frac{-\infty}{0}$

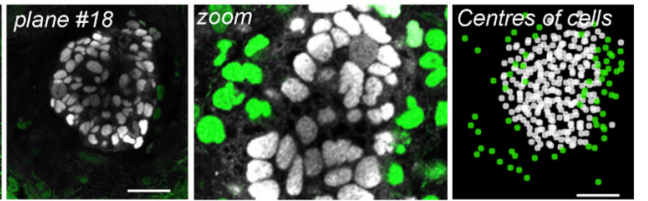

C PAR6

CK7

F-actin/DAPI

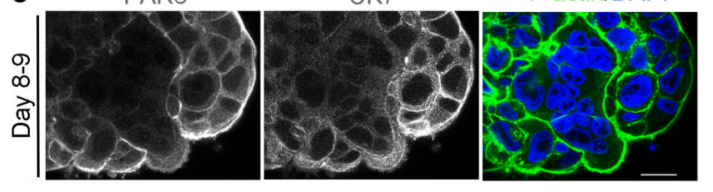

e

OCT4/actin/DAPI 3D reconstruction

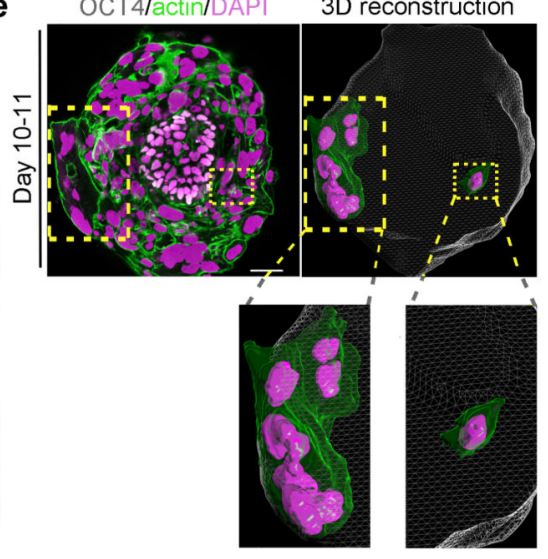

Figure 3. Analysis of embryonic and extra-embryonic lineages in human embryos cultured through implantation stages in vitro.

Human embryos developing in vitro until day 11 were fixed and stained at indicated time points. a, Representative confocal Z sections of human embryos stained for OCT4 and GATA6. Right panels show the centre of all OCT4-expressing (white) and GATA6expressing (green) cells. All positive cells were counted regardless of the fluorescence intensity value. $\mathbf{b}$, Representative confocal $\mathrm{Z}$ section of a day 9-10 embryo stained for OCT4, GATA6 and CK7. c, Representative confocal Z section of a day 8-9 embryo stained 
for PAR6 and CK7. d, Representative confocal Z section of human embryos stained for Factin and DAPI. Arrowheads point to multinucleated cells. e, 3D reconstruction of the cellular and nuclear shape of representative trophectoderm cells. Note that cells in close proximity to the epiblast have a single nucleus, whereas cells in the periphery of the embryo are multinucleated. All yellow squares indicate the regions in the embryos that are shown with higher magnification. All scale bars, $50 \mu \mathrm{m}$. These data involved the assessment of a total of 59 embryos collected across 6 experiments, out of which 29 showed preservation of the embryonic lineage. 
a

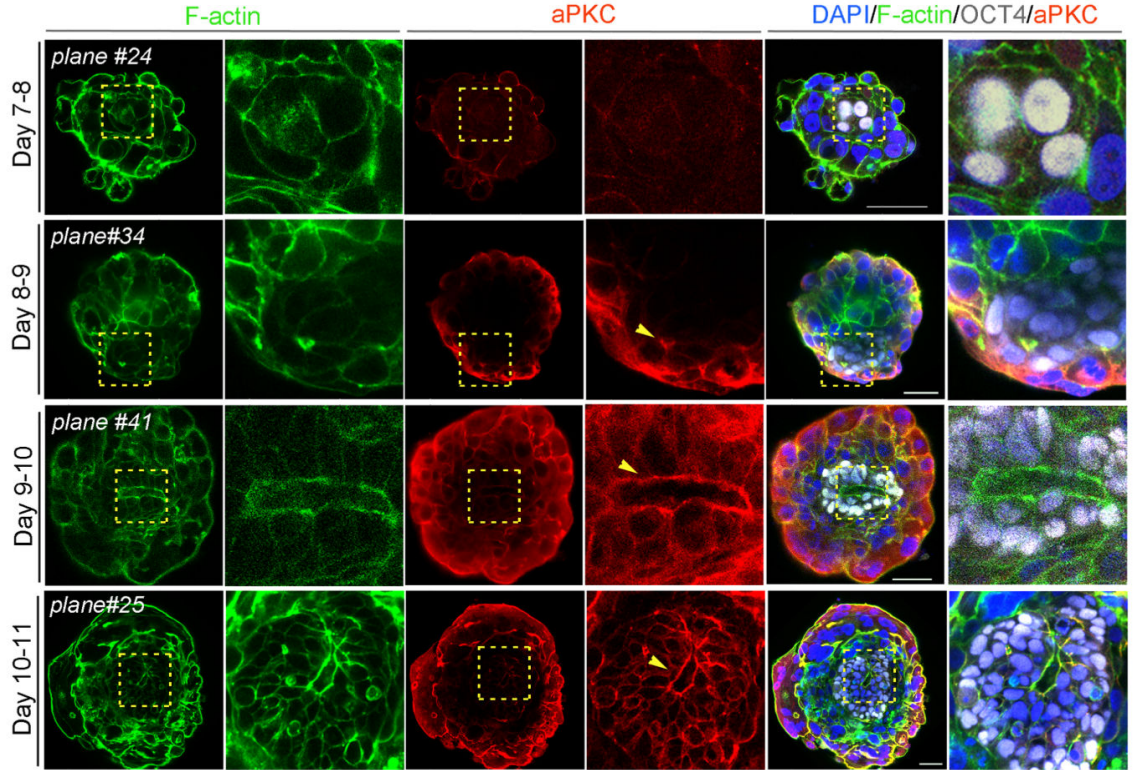

b

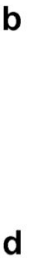

d
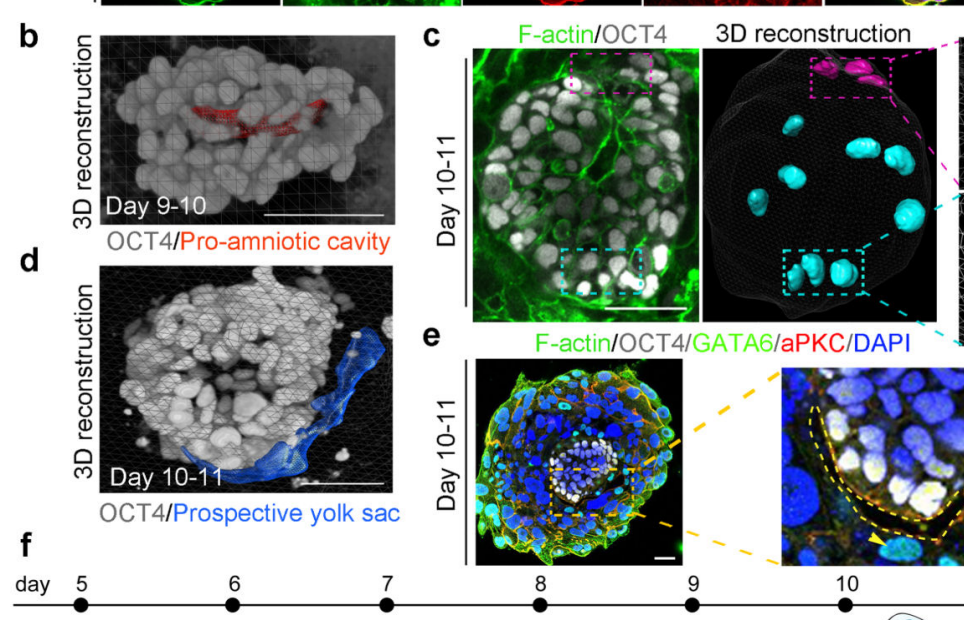

F-actin/OCT4/GATA6/aPKC/DAP
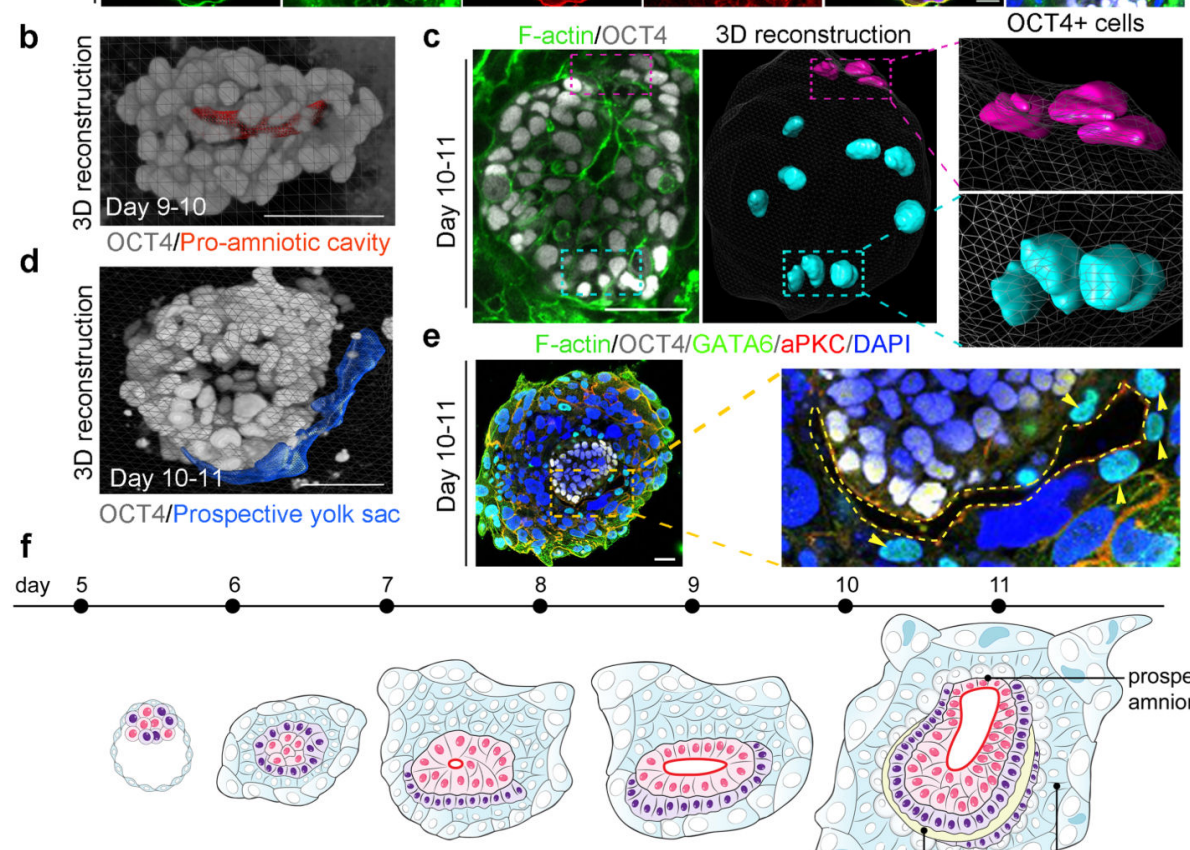

$\square$ EPI (OCT4) $\square$ hypoblast (GATA6) $\square$ TE (CK7) $\square$ proamniotic cavity (aPKC) $\square$ lacuna

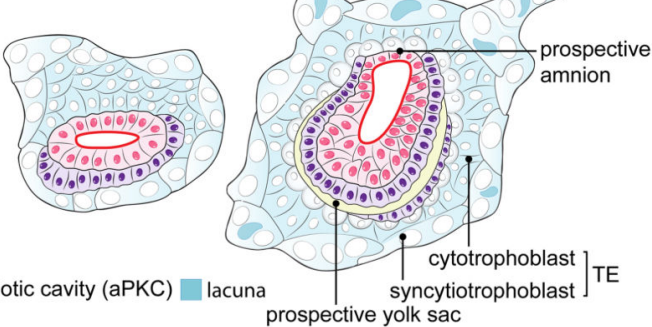

Figure 4. Remodelling of the epiblast in human embryos cultured through implantation stages. Human embryos developing in vitro until day 11 were fixed and stained at indicated time points. a, Representative confocal Z sections of human embryos stained for F-actin, aPKC, and OCT4. Arrowheads indicate the incipient pro-amniotic cavity. $\mathbf{b}, 3 \mathrm{D}$ reconstruction of the pro-amniotic cavity (shown in red). The nuclei of OCT4-expressing epiblast cells is shown in gray. $\mathbf{c}, 3 \mathrm{D}$ reconstruction of the cellular shape of representative OCT4-expressing epiblast cells. d, 3D reconstruction of the prospective yolk sac (shown in blue). The nuclei of OCT4-expressing epiblast cells is shown in gray. e, Day 10-11 embryo stained for F-actin, 
OCT4, GATA6 and aPKC. Note the presence of GATA6-expressing cells on both sides of the cavity (arrowheads). The prospective yolk sac is indicated with a dashed line. f, Model of human embryo implantation morphogenesis based on our results and the Carnegie Series. The major remodelling events that take place during this transition are: (i) segregation of epiblast and hypoblast progenitors -day 7; (ii) polarisation and pro-amniotic cavity formation in the epiblast -day 8-10; (iii) differentiation of the trophectoderm into cytotrophoblast and syncytiotrophoblast cells -day 8-10; (iv) formation of the prospective amniotic epithelium, the prospective yolk sac and the bilaminar disc-day 10-11. All squares indicate the regions in the embryos that are shown with higher magnification. All scale bars, $50 \mu \mathrm{m}$. These data involved the assessment of a total of 59 embryos collected across 6 experiments, out of which 9 showed pro-amniotic cavity formation. 

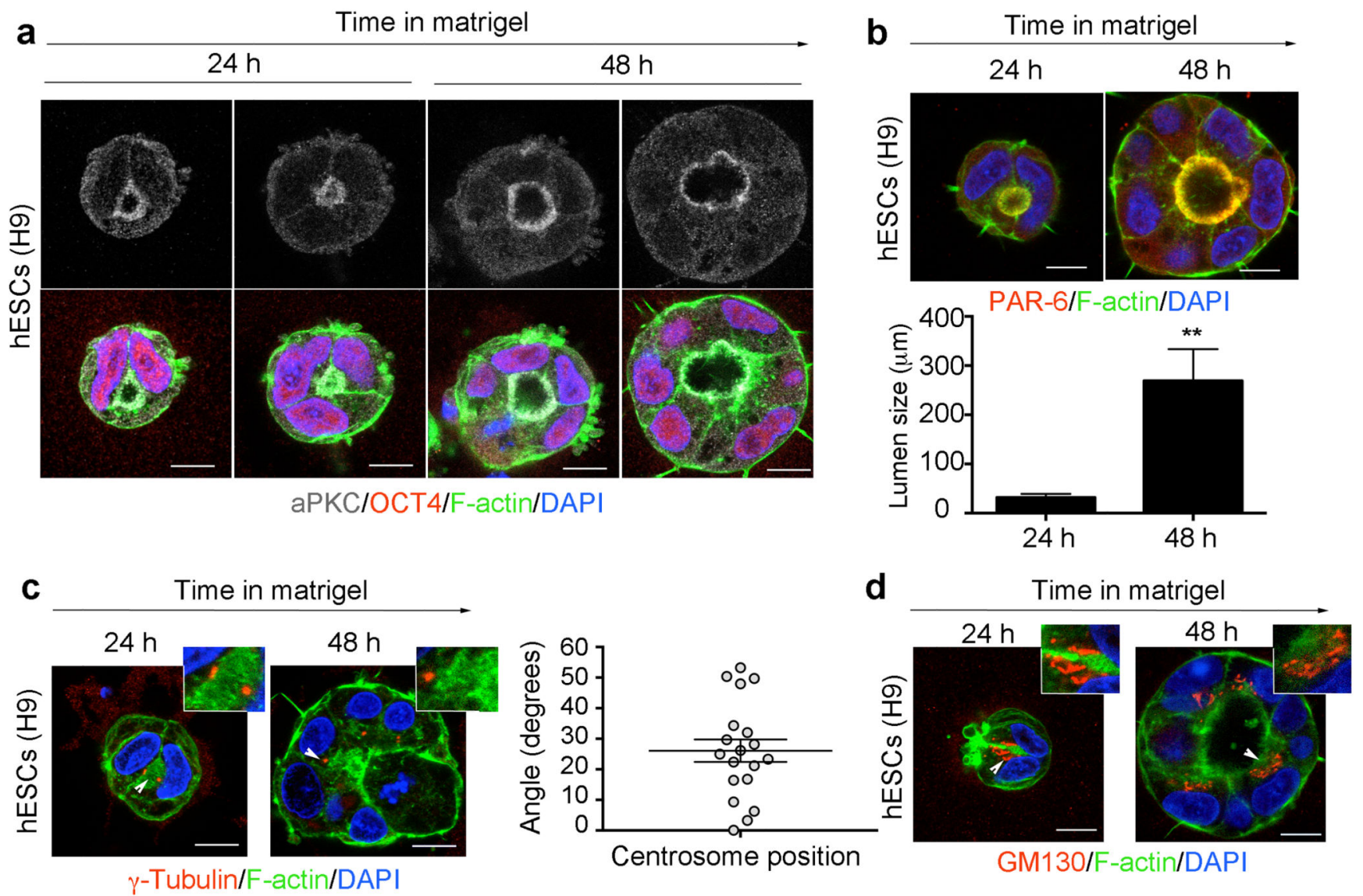

Figure 5. Self-organisation of hESCs in response to extracellular matrix signaling. hESCs were plated in a 3D matrix of matrigel and analysed at the indicated time points. a, hESCs stained for OCT4 as a marker of pluripotency, aPKC as marker of apical polarisation and F-actin to reveal the general organisation of the cells. b, hESCs stained for PAR-6 and F-actin. The size of the lumen was quantified. Data is shown as average \pm s.e.m. Note the increase in lumen size with time ( $\mathrm{n}=10 \mathrm{hESC}$ organoids per time point). Unpaired two-tailed Student's t test with Welch's correction, $* * \mathrm{p}=0.0049$. c, hESCs stained for the centrosome marker $\boldsymbol{\gamma}$-Tubulin and F-actin. Arrowheads indicate the polarised apical localization of the centrosome. The nucleus centrosome angle with respect to the nucleus-nucleus axis is shown. Each dot represents an individual centrosome. Data is shown as average \pm s.e.m. ( $n=19$ centrosomes). d, hESCs stained for the Golgi marker GM130 and F-actin.

Arrowheads indicate the polarised apical localization of the Golgi. All scale bars, $10 \mu \mathrm{m}$. These data involved the assessment of a minimum of $10 \mathrm{hESC}$ organoids per panel, all of which showed the indicated phenotype. Images are representative of a minimum of 2 independent experiments per panel. 
a

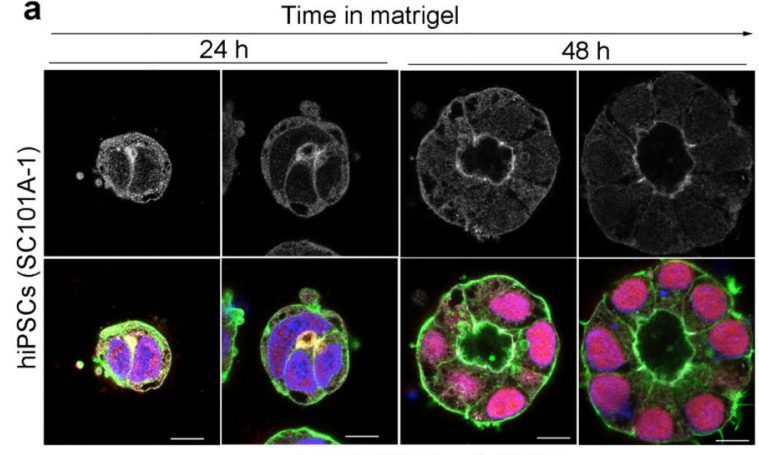

aPKC/OCT4/F-actin/DAPI

b

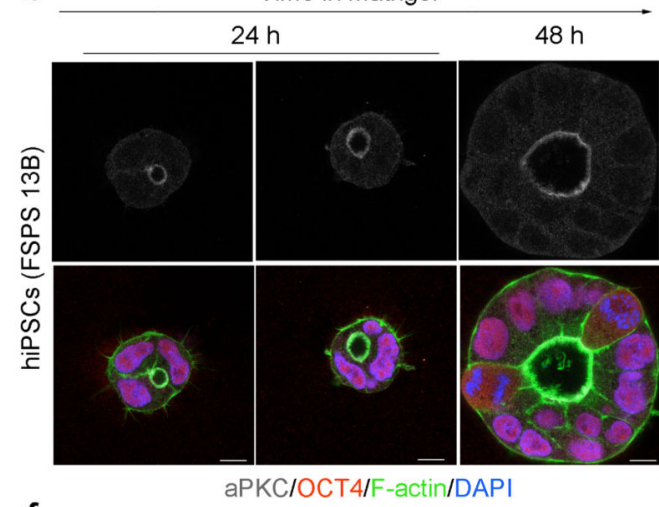

f

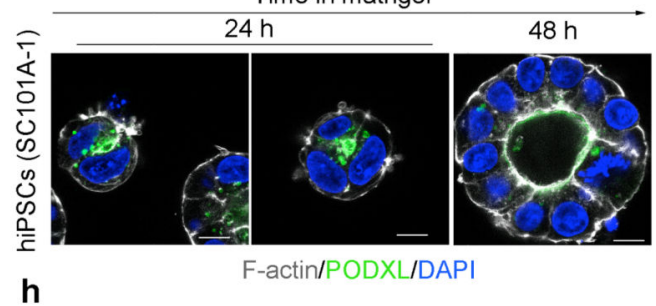

h

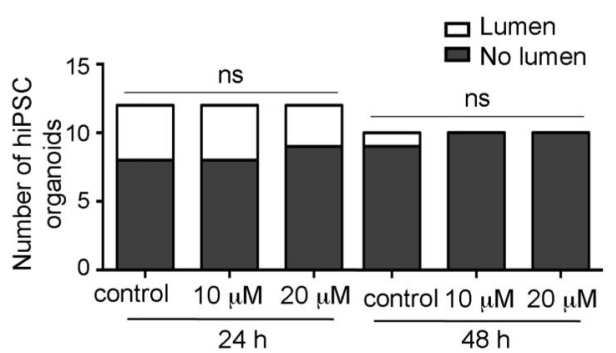

C

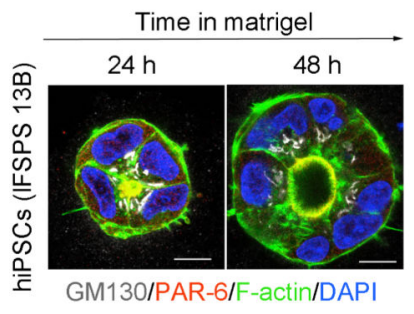

d

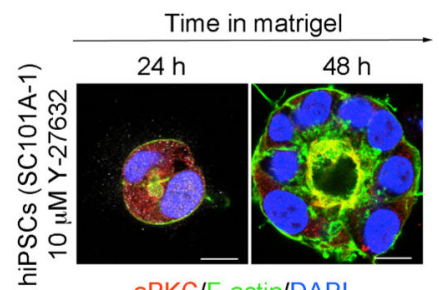

e

aPKC/F-actin/DAPI

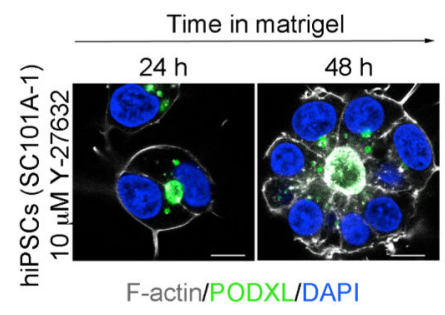

g

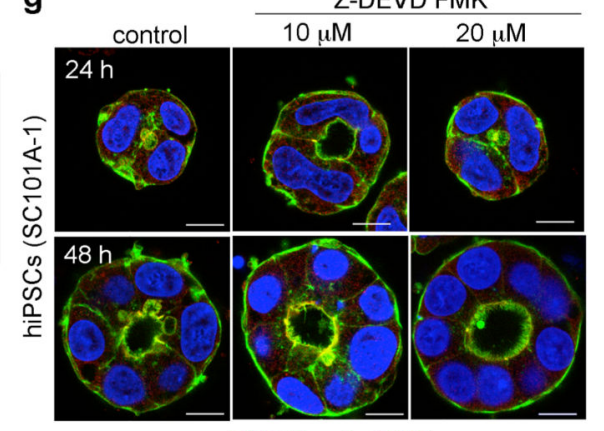

aPKC/F-actin/DAPI

Figure 6. Self-organisation of hiPSCs in response to extracellular matrix signaling.

hiPSCs were plated in a 3D matrix of matrigel and analysed at the indicated time points. ab, hiPSCs stained for aPKC, OCT-4 and F-actin. c, hiPSCs stained for PAR-6, GM130 and F-actin. d, hiPSCs cultured in the presence of the ROCK inhibitor Y-27632 and stained for aPKC and F-actin. e-f, hiPSCs cultured in the presence (e) or absence (f) of ROCK inhibitor, and stained for PODXL and F-actin $\mathbf{g}$, hiPSCs were treated with the caspase 3 inhibitor ZDEVD FMK and lumen formation was analysed 24 and 48 hours after plating by staining for aPKC and F-actin. h, Quantification of lumen formation in the presence of Z-DEVD 
FMK. Data is shown as a contingency table. Fisher's exact test. ns: not significant ( $\mathrm{n}=12$ hiPSC organoids per condition $-24 \mathrm{~h}$ time point-; $\mathrm{n}=10 \mathrm{hiPSC}$ organoids per condition $-48 \mathrm{~h}$ time point-). Data presented in this figure involved the assessment of a minimum of 10 hESC organoids per panel and per condition across a minimum of 2 independent experiments per panel. 